\title{
POINTWISE BOUNDED APPROXIMATION AND HYPODIRICHLET ALGEBRAS 1
}

\author{
BY T. W. GAMELIN ${ }^{2}$ AND JOHN GARNETT \\ Communicated by F. W. Gehring, July 2, 1970
}

1. Introduction. Let $U$ be an open subset of the Riemann sphere $S^{2}$. The algebra of bounded analytic functions on $U$ is denoted by $H^{\infty}(U)$ and the algebra of continuous functions on $\bar{U}$ which are analytic on $U$ is denoted by $A(U)$. We are interested in the following two questions:

(1) When does $\operatorname{Re} A(U)$ have finite defect in $C_{R}(\partial U)$ ? That is, when does the uniform closure of the real parts of functions in $A(U)$ have finite codimension in the space of continuous real-valued functions on the boundary $\partial U$ of $U$ ?

(2) When is $A(U)$ pointwise boundedly dense in $H^{\infty}(U)$ ? That is, when can every function in $H^{\infty}(U)$ be approximated pointwise on $U$ by a bounded sequence in $A(U)$ ?

These two problems are related by the following theorem, which extends a result of A. M. Davie [2].

Theorem 1. Suppose $\partial U$ has no isolated points. Then $\operatorname{Re} A(U)$ has finite defect in $C_{R}(\partial U)$ if and only if $S^{2} \backslash U$ has a finite number of components and $A(U)$ is pointwise boundedly dense in $H^{\infty}(U)$.

In this announcement, we wish to elaborate on this result, and to state answers to questions (1) and (2) in terms of analytic capacity.

2. A theorem on uniform approximation. By $K$ we will always denote a compact subset of the complex plane. The algebra $R(K)$ is the uniform closure on $K$ of the rational functions with poles off $K$. The algebra $A(K)$ consists of the continuous functions on $K$ which are analytic on the interior $K^{\circ}$ of $K$. With this notation, $A(K)$ consists of the functions in $A\left(K^{\circ}\right)$, extended in all possible continuous ways to $K$. Consequently we can deduce from each theorem about $A(U)$ a corresponding theorem about $A(K)$, by setting $U=K^{\circ}$. In

AMS 1970 subject classifications. Primary 41A20, 41A30, 46J10.

Key words and phrases. Pointwise bounded approximation, uniform approximation, rational functions, analytic capacity, dirichlet algebras.

1 The preparation of this announcement was sponsored in part by NSF Grant GP-11475.

2 Fellow of the Alfred P. Sloan Foundation.

Copyright (a) 1971, American Mathematical Soclety 
turn, the theorems we will state for $R(K)$ will all follow from the version for $A(K)$ and the following result, which extends Theorem 3.4 of $[4]$.

THEOREM 2. If (i) $R(\partial K)=C(\partial K)$, and (ii) $R(K)$ is pointwise boundedly dense in $H^{\infty}\left(K^{\circ}\right)$, then $R(K)=A(K)$.

Condition (i) is necessary, but condition (ii) is not. It would be of interest to relax condition (ii).

3. Pointwise bounded approximation. Necessary and sufficient conditions for the pointwise bounded density of $A(U)$ in $H^{\infty}(U)$ were given in [4], and were sharpened by Davie in [2]. In order to state a further strengthening of this characterization, we introduce more definitions.

A curvilinear null set is a subset of zero outer length lying on a twice continuously differentiable curve. A $\sigma$-curvilinear null set is a countable union of curvilinear null sets. The analytic capacity of a planar set $S$ is

$\gamma(S)=\sup \left\{\left|f^{\prime}(\infty)\right|: f\right.$ is analytic off some compact subset

$$
\text { of } S,|f| \leqq 1\} \text {. }
$$

The continuous analytic capacity of a set $S$ is

$$
\alpha(S)=\sup \left\{\left|f^{\prime}(\infty)\right|: f \text { as above, } f \in C\left(S^{2}\right)\right\} .
$$

The open disc centered at $z_{0}$ with radius $\delta$ is denoted by $\Delta\left(z_{0} ; \delta\right)$.

THEOREM 3. The following are equivalent:

(i) $A(U)$ is pointwise boundedly dense in $H^{\infty}(U)$.

(ii) $\gamma(D \backslash U)=\alpha(D \backslash U)$ for each bounded open set $D$.

(iii) There is a $\sigma$-curvilinear null set $E$ such that for each $z \in(\partial U) \backslash E$, $z \neq \infty$, there exists $r \geqq 1$ satisfying

$$
\liminf _{\delta \rightarrow 0} \frac{\alpha(\Delta(z ; r \delta) \backslash U)}{\gamma(\Delta(z ; \delta) \cap \partial U)}>0 .
$$

Theorem 3 is analogous to Vitushkin's Theorem (VIII. 8.2 of [3]) characterizing those $K$ for which $R(K)=A(K)$. An additional difficulty with pointwise bounded approximation is that one does not automatically get good bounds on the norms of approximating sequences. The proofs of both the necessity of (ii) and the sufficiency of (iii) depend not only on Vitushkin's constructive techniques, but also on the abstract tools of functional analysis, and especially on an argument involving the separation theorem for convex sets which stems from Ahern and Sarason [1]. 
Using Theorem 2, one obtains the following companion theorem for $R(K)$.

THEOREM 4. The following are equivalent:

(i) $R(\partial K)=C(\partial K)$, and $R(K)$ is pointwise boundedly dense in $H^{\infty}\left(K^{\circ}\right)$.

(ii) $\gamma\left(D \backslash K^{\circ}\right)=\gamma(D \backslash K)$ for each bounded open set $D$.

(iii) There is a $\sigma$-curvilinear null set $E$ such that for each $z \in(\partial K) \backslash E$, there exists $r \geqq 1$ satisfying

$$
\liminf _{\delta \rightarrow 0} \frac{\gamma(\Delta(z ; r \delta) \backslash K)}{\gamma\left(\Delta(z ; \delta) \cap \partial K^{\circ}\right)}>0 .
$$

4. Algebras with finite real defect. Recall that $A(U)$ is dirichlet on $\partial U$ if $\operatorname{Re} A(U)$ is dense in $C_{R}(\partial U)$. Also, $A(U)$ is hypodirichlet if the defect of $\operatorname{Re} A(U)$ in $C_{R}(\partial U)$ is finite, while the linear span of $\log \left|A(U)^{-1}\right|$ is dense in $C_{R}(\partial U)$.

Theorem 1 can be restated in the following form, with the aid of Theorem 3 .

TheOREM 5. Suppose that $S^{2} \backslash U$ has a finite number of components, none of which are points. Then the following are equivalent:

(i) Each $z \in U$ has a finite dimensional set of representing measures (on $\partial U$, for $A(U)$ ).

(ii) $\operatorname{Re} A(U)$ has finite defect in $C_{R}(\partial U)$.

(iii) If $Y$ is any component of $S^{2} \backslash U$ and $V=S^{2} \backslash Y$, then $A(V)$ is a dirichlet algebra on $\partial \mathrm{V}$.

(iv) $A(U)$ is pointwise boundedly dense in $H^{\infty}(U)$.

(v) If $\delta>0$ is sufficiently small, then

$$
\alpha(\Delta(z ; \delta) \backslash U) \geqq \delta / 4, \quad z \in \partial U .
$$

(vi) There is a $\sigma$-curvilinear null set $E$ such that for all $z \in(\partial U) \backslash E$,

$$
\liminf _{\delta \rightarrow 0} \frac{\alpha(\Delta(z ; \delta) \backslash U)}{\delta}>0
$$

Under the equivalent conditions of Theorem 5, we can describe explicitly the real functionals on $C(\partial U)$ which are orthogonal to $A(U)$. Indeed, suppose there are $r$ components $E_{1}, \cdots, E_{r}$ of $S^{2} \backslash U$. Let $\gamma_{j}$ be a cycle in $U$ which surrounds $E_{j}$ in the usual sense of contour integration. Under our hypotheses, every function $u \in C_{R}(\partial U)$ has a continuous extension $\tilde{u}$ to $\bar{U}$ harmonic on $U$. Define $L_{j}(u)$ to be the increment of the harmonic conjugation function of $\tilde{u}$ along $\boldsymbol{\gamma}_{j}$. Then the functionals $u \rightarrow L_{j}(u)$ are continuous real functionals on 
$C_{R}(\partial U)$ which are orthogonal to $A(U)$, and $L_{2}, \cdots, L_{\mathrm{r}}$ form a basis for the space of real functionals orthogonal to $A(U)$. In particular the defect of $\operatorname{Re} A(U)$ in $C_{R}(\partial U)$ is equal to $r-1$.

Now suppose the first $s$ of the $E_{j}$ have interior while the remaining $r-s$ do not. Let $q_{j} \in E_{j}^{\circ}, 1 \leqq j \leqq s$. Then the linear span of $\log \left|A(U)^{-1}\right|$ has defect $r-s(r-1$, if $s=0)$ in $C_{r}(\partial U)$. The closed linear span of $\log \left|A(U)^{-1}\right|$ coincides with the closed linear span of $\operatorname{Re} A(U)$ and the functions $\log \left|\left(z-q_{j}\right) /\left(z-q_{1}\right)\right|, 2 \leqq j \leqq s$.

The corresponding theorems for $A(K)$ and $R(K)$ are as follows.

THEOREM 6. Suppose $S^{2} \backslash K^{\circ}$ has only a finite number of components. Then the following are equivalent:

(i) Each $z \in K^{\circ}$ has a finite dimensional set of representing measures (on $\partial K$, for $A(K)$ ).

(ii) $\operatorname{Re} A(K)$ has finite defect in $C_{R}(\partial K)$.

(iii) $A(K)$ is a hypodirichlet algebra.

(iv) $A(K)$ is pointroise boundedly dense in $H^{\infty}\left(K^{\circ}\right)$.

(v) If $\delta>0$ is sufficiently small, then

$$
\alpha\left(\Delta(z ; \delta) \backslash K^{\circ}\right) \geqq \delta / 4, \quad z \in \partial K .
$$

(vi) There is a $\sigma$-curvilinear null set $E$ such that for all $z \in(\partial K) \backslash E$,

$$
\liminf _{\delta \rightarrow 0} \frac{\alpha\left(\Delta(z ; \delta) \backslash K^{\circ}\right)}{\delta}>0 .
$$

When finite, the defect of $\operatorname{Re} A(K)$ in $C_{R}(\partial K)$ is equal to $r-1$, wherer is the number of components of $S^{2} \backslash K^{\circ}$. If furthermore $q_{1}, \cdots, q_{r-1} b e-$ long to the interiors of distinct components of $S^{2} \backslash K^{\circ}$, then the linear span of $\operatorname{Re} A(K)$ and the functions $\log \left|z-q_{1}\right|, \cdots, \log \left|z-q_{r-1}\right|$ is dense in $C_{R}(\partial K)$.

THEOREM 7. Suppose $S^{2} \backslash K^{\circ}$ has only a finite number of components. Then the following are equivalent:

(i) Each $z \in K^{\circ}$ has a finite dimensional set of representing measures (on $\partial K$, for $R(K)$ ).

(ii) $\operatorname{Re} R(K)$ has finite defect in $C_{R}(\partial K)$.

(iii) $R(K)$ is a hypodirichlet algebra.

(iv) $R(K)$ is pointwise boundedly dense in $H^{\infty}\left(K^{\circ}\right)$, and $R(\partial K)$ $=C(\partial K)$.

(v) If $\delta>0$ is sufficiently small, then

$$
\gamma(\Delta(z ; \delta) \backslash K) \geqq \delta / 4, \quad z \in \partial K .
$$

(vi) There is a $\sigma$-curvilinear null set $E$ such that for all $\mathrm{z} \in(\partial K) \backslash E$, 


$$
\liminf _{\delta \rightarrow 0} \frac{\gamma(\Delta(z ; \delta) \backslash K)}{\delta}>0
$$

When finite, the defect of $\operatorname{Re} R(K)$ is equal to $r-1(0$, if $r=0)$, where $r$ is the number of components of $S^{2} \backslash K^{\circ}$.

It is easy to formulate geometric criteria which ensure the validity of the estimate (vi). Using the fact that the analytic capacity of a continuum is comparable to its diameter, we can state the following corollary. Recall that the inner boundary of $K$ consists of those points $z \in \partial K$ which do not lie on the boundary of one of the components of $S^{2} \backslash K$.

THEOREM 8. If $\partial K$ is connected, and the inner boundary of $K$ is a $\sigma$ curvilinear null set, then $R(K)$ is pointwise boundedly dense in $H^{\infty}\left(K^{\circ}\right)$, and $R(K)$ is a dirichlet algebra.

When $K$ has a connected complement, the first and second conclusions of Theorem 8 reduce respectively to the Farrell-RubelShields Theorem (VI.5.1 of [3]) on pointwise bounded approximation, and the Walsh-Lebesgue Theorem (II.3.3 of [3]) on uniform approximation by harmonic polynomials in $x$ and $y$. When the complement of $K$ has a finite number of components, we find ourselves in the situation treated by Ahern and Sarason [1]. The theorem, in the case in which the inner boundary is at most countable, was obtained by McCullough [7].

Proofs and further applications of these results in the case of dirichlet algebras will appear in [5].

\section{REFERENCES}

1. P. R. Ahern and D. Sarason, On some hypo-Dirichlet algebras of analytic functions, Amer. J. Math. 89 (1967), 932-941. MR 36 \#4338.

2. A. M. Davie, Bounded approximation and Dirichlet sets, J. Functional Analysis (to appear).

3. T. Gamelin, Uniform algebras, Prentice-Hall, Englewood Cliffs, N. J., 1969.

4. T. Gamelin and J. Garnett, Constructive techniques in rational approximation, Trans. Amer. Math. Soc. 143 (1969), 187-200.

5. ——, Pointwise bounded approximation and Dirichlet algebras, J. Functional Analysis (to appear).

6. I. Glicksberg, Dominant representing measures and rational approximation, Trans. Amer. Math. Soc. 130 (1968), 425-462. MR 37 \#744.

7. T. A. McCullough, Rational approximation on certain plane sets, Pacific J. Math. 29 (1969), 631-640. MR 40 \#374.

8. A. G. Vituskin, Analytic capacity of sets in problems of approximation theory, Uspehi Mat. Nauk 22 (1967), no. 6 (138), 141-199 = Russian Math. Surveys 22 (1967), 139-200. MR 37 \#5404.

University of California, los Angeles, California 90024 


\title{
THE HOMOLOGY OF THE CLASSICAL GROUPS OVER THE DYER-LASHOF ALGEBRA ${ }^{1,2}$
}

\author{
BY STANLEY O. KOCHMAN
}

Communicated by N. E. Steenrod, July 1, 1970

1. Introduction. By Bott periodicity the classical groups, their classifying spaces, their homogeneous spaces, $\operatorname{Im} J$ and $B \operatorname{Im} J$ are infinite loop spaces, and hence the Dyer-Lashof operations act on their $\bmod p$ homology for $p$ a prime number. We will list the basic properties of the Dyer-Lashof operations in $\S 2$. These operations have been calculated in the homology of all these spaces, and the dual operations have also been computed. As applications one can calculate the indecomposable elements of the homology of these spaces over the Dyer-Lashof algebra R and the AR-Hopf algebra maps between any two classical groups or any two of their classifying spaces. In this paper we will summarize our results for $B U, \mathrm{BU}$ (the connected and infinite component versions of the classifying space of the infinite unitary group), $O, S O, B \operatorname{Im} J$ and $\operatorname{Im} J$.

These results have been applied to the study of $H_{*}(F)$ and $H_{*}(B F)$ by J. P. May [9] and I. Madsen [7]. They are also useful in cobordism theory (see for example T. tom Dieck [5, p. 396]).

The author is very grateful to J. Peter May for his guidance, suggestions and generosity with his time throughout the preparation of this paper.

Notation. All elements of a graded object will be indexed by their degree with the exception of the Chern and Wu classes. All homology and cohomology will have $Z_{p}$ coefficients for $p$ a prime number. When a result differs for $p$ an odd prime and $p=2$ then the result for $p=2$ will be placed in square brackets.

2. The Dyer-Lashof operations. The homology of an infinite loop space $B$ has natural homomorphisms $Q^{i}: H_{*}(B) \rightarrow H_{*}(B)$ for $i \geqq 0$ of degree $2 i(p-1)$ [of degree $i$ ] which have been studied by S. Araki and

AMS 1970 subject classifications. Primary 55F45; Secondary 55G99.

Key words and phrases. Classical groups, classifying space, infinite loop space, Dyer-Lashof algebra, Bott periodicity, suspension, Nishida relations, image of J.

1 During the preparation of this paper the author was supported by a National Science Foundation Graduate Fellowship.

2 The work announced here is contained in the author's doctoral thesis, submitted to the University of Chicago. 
T. Kudo [3], W. Browder [4], E. Dyer and R. Lashof [6], G. Nishida [10] and J. P. May [8]. Theorem 1 summarizes some of their results.

THEOREM 1. The Dyer-Lashof operations satisfy the following propperties:

(a) $Q^{0}(\phi)=\phi$ and $Q^{i}(\phi)=0$ if $i>0$ where $\phi \in H_{0}(B)$ is the identity element for the loop product in $H_{*}(B)$.

(b) $Q^{i}(x)=0$ if $2 i<\operatorname{deg} x$ [if $\left.i<\operatorname{deg} x\right]$.

(c) $Q^{i}(x)=x^{p}$ if $2 i=\operatorname{deg} x[$ if $i=\operatorname{deg} x]$.

(d) $\sigma_{*} \circ Q^{i}=Q^{i} \circ \sigma_{*}$ where $\sigma_{*}: I H_{*}(\Omega B) \rightarrow H_{*}(B)$ is the homology suspension map.

(e) (Multiplicative Cartan formula) $Q^{r}(x y)=\sum_{i=0}^{r} Q^{i}(x) Q^{r-i}(y)$.

(f) (Comultiplicative Cartan formula)

$$
\psi \circ Q^{r}(x)=\sum_{i=0}^{r} \sum Q^{i}\left(x^{\prime}\right) \otimes Q^{r-i}\left(x^{\prime \prime}\right)
$$

where $\psi(x)=\sum x^{\prime} \otimes x^{\prime \prime}$.

(g) $\chi \circ Q^{i}=Q^{i} \bigcirc \chi$ where $\chi$ is the conjugation of $H_{*}(B)$.

(h) (Nishida relations) Let $P_{*}^{s}: H_{*}(B) \rightarrow H_{*}(B)$ of degree $-2 s(p-1)$ [of degree $-s$ ] be dual to the Steenrod operation $P^{s}$. Then

$$
P_{*}^{s} \circ Q^{r}=\sum(-1)^{i+s}(s-p i, r(p-1)-p s+p i) Q^{r-s+i} \circ P_{*}^{i} .
$$

We use the convention $(i, j)=(i+j) ! / i ! j !$ if $i \geqq 0$ and $j \geqq 0$ while $(i, j)=0$ if $i<0$ or $j<0$. Thus the above sum is taken over all integers $i$.

(i) (Adem relations) If $a>p b$ then

$$
Q^{a} \circ Q^{b}=\sum(-1)^{a+i}(p i-a, a-(p-1) b-i-1) Q^{a+b-i} \circ Q^{i} .
$$

Definition 2. The Dyer-Lashof algebra $R$ is the quotient algebra $F / J$ where $F$ is the free associative algebra generated by $\left\{Q^{r}, \beta Q^{r+1} \mid r \geqq 0\right\}$ [by $\left\{Q^{r} \mid r \geqq 0\right\}$ ], and $J$ is the ideal of $F$ consisting of all elements which annihilate every element of every infinite loop space.

3. Results on $B U$ and BU. Recall that $H^{*}(B U)=P\left\{c_{n} \mid n \geqq 1\right\}$ as algebras where $c_{n}$ is the Chern class of degree $2 n, c_{0}=1$ and $\psi\left(c_{n}\right)$ $=\sum_{i=0}^{n} c_{i} \otimes c_{n-i}$. Let $a_{2 n}=\left(c_{1}^{n}\right)^{*}$ and $\mathrm{p}_{2 n}=c_{n}^{*}$ in the dual basis of the basis of $H^{*}(B U)$ which consists of monomials in the Chern classes. Then $H_{*}(B U)=P\left\{a_{2 n} \mid n \geqq 1\right\}$ as algebras with $\psi\left(a_{2 n}\right)$ $=\sum_{i=0}^{n} a_{2 i} \otimes a_{2 n-2 i}$, and $\left\{\mathrm{p}_{2 n} \mid n \geqq 1\right\}$ is a basis for the primitive elements of $H_{*}(B U)$. For $r \geqq 0$ we have the duals of the Dyer-Lashof operations $Q_{*}^{r}: H^{*}(B U) \rightarrow H^{*}(B U)$ of degree $-2 r(p-1)$ [of degree 
$-r$. Theorems 3 and 4 are our main results on $B U$.

THEOREM 3. In $H_{*}(B U)$ for $r \geqq 0$ and $n \geqq 1$,

$$
\begin{aligned}
Q^{r}\left(\mathrm{p}_{2 n}\right) & =(-1)^{r+n}(n-1, r-n) \mathrm{p}_{2 n+2 r(p-1)} \\
{\left[Q^{2 r}\left(\mathrm{p}_{2 n}\right)\right.} & \left.=(n-1, r-n) \mathrm{p}_{2 n+2 r}\right],
\end{aligned}
$$

and

$$
\begin{aligned}
Q^{r}\left(a_{2 n}\right) & =(-1)^{r+n+1}(n, r-n-1) a_{2 n+2 r(p-1)}+\text { decomposables } \\
{\left[Q^{2 r}\left(a_{2 n}\right)\right.} & \left.=(n, r-n-1) a_{2 n+2 r}+\text { decomposables }\right] .
\end{aligned}
$$

THEOREM 4. In $H^{*}(B U)$ for $r \geqq 0$ and $n \geqq 1$,

$$
\begin{aligned}
Q_{*}^{r}\left(c_{n}\right) & =(-1)^{r+n}(n-r(p-1)-1, p r-n) c_{n-r(p-1)} \\
{\left[Q_{*}^{2 r}\left(c_{n}\right)\right.} & \left.=(n-r-1,2 r-n) c_{n-r}\right] .
\end{aligned}
$$

Theorem 3 is proved by using the comultiplicative Cartan formula, the Nishida relations and Bott periodicity. An algorithm can then be given for computing $Q^{r}\left(a_{2 n}\right)\left[Q^{2 r}\left(a_{2 n}\right)\right]$ by induction on $n+r(p-1)$ and for fixed $n+r(p-1)$ by induction on $n$ using the following seven properties that the $Q^{r}$ satisfy on $H_{*}(B U):(\mathrm{a}),(\mathrm{b}),(\mathrm{c}),(\mathrm{e}),(\mathrm{f}),(\mathrm{h})$ of Theorem 1 and Theorem 3 . Theorem 4 is now proved by defining homomorphisms $R_{*}^{s}$ on $H^{*}(B U)$ by

$$
\begin{aligned}
R_{*}^{s}\left(c_{n}\right) & =(-1)^{s+n}(n-s(p-1)-1, p s-n) c_{n-s(p-1)} \\
{\left[R_{*}^{2 s}\left(c_{n}\right)\right.} & \left.=(n-s-1,2 s-n) c_{n-s}\right]
\end{aligned}
$$

and insisting that the $R_{*}^{s}$ satisfy the multiplicative Cartan formula. Then the dual maps $R^{8}$ can be shown to satisfy the above seven properties. Hence $R^{s}=Q^{s}$ for all $s \geqq 0$ by the algorithm for computing the Dyer-Lashof operations on $H_{*}(B U)$.

Recall that $H_{*}(\mathrm{BU})=H_{*}(B U) \otimes Z_{p}(Z)$ as Hopf algebras where $Z_{p}(Z)$ is the group algebra of the integers over $Z_{p}$. Elements of $H_{*}(\mathrm{BU})$ are written $x \otimes[i]$ for $x \in H_{*}(B U)$ and $i \in Z \subset Z_{p}(Z)$. Since the canonical inclusion $B U \rightarrow B U$ induces the map $x \rightarrow x \otimes[0]$ in homology for $x \in H_{*}(B U)$, the following theorem together with our knowledge of the Dyer-Lashof operations on $H_{*}(B U)$ tell us how the Dyer-Lashof operations act on $H_{*}(\mathrm{BU})$.

THEOREM 5. In $H_{*}(\mathrm{BU})$ for $n \geqq 1, Q^{n}(1 \otimes[1])=\gamma_{n}\left(\mathrm{p}_{2(p-1)}\right) \otimes[p]$ $\left[Q^{2 n}(1 \otimes[1])=a_{2 n} \otimes[2]\right]$ where $\gamma_{n}\left(\mathrm{p}_{2(p-1)}\right)$ is $\left(c_{p-1}^{n}\right)^{*}$ in the dual basis of the basis of $H^{*}(B U)$ which consists of monomials in the Chern classes. 